\title{
Uma plataforma tecnológica para o ensino de engenharia de controle
}

\author{
Adelson Siqueira Carvalho, UFRGS, adelsonsc@yahoo.com.br \\ Dante Augusto Couto Barone, UFRGS, barone@inf.ufrgs.br \\ Milton Antônio Zaro, UFRGS, zaro@ufrgs.br
}

\begin{abstract}
Resumo: Este trabalho apresenta uma plataforma tecnológica como recurso didático para o ensino de engenharia de controle. A plataforma em questão combina o potencial educacional do software de projeto e simulação de sistemas de controle - MATLAB® e a conexão deste software a uma coluna de destilação piloto. A hipótese de pesquisa é que a utilização de plataformas tecnológicas como esta ajudam a desenvolver aspectos relevantes não estimulados apenas com simulação. Para tanto, um experimento baseado na teoria construtivista e sócio-interacionista foi concebido para detectar a evolução dos alunos e captar suas impressões sobre plataforma. Os resultados são apresentados na forma de gráficos e relatos das impressões do professor.
\end{abstract}

Palavras chave: plataforma tecnológica, sistemas de controle, teoria construtivista.

\section{A technological platform for teaching control engineering}

\author{
Adelson Siqueira Carvalho, UFRGS, adelsonsc@yahoo.com.br \\ Dante Augusto Couto Barone, UFRGS, barone@inf.ufrgs.br \\ Milton Antônio Zaro, UFRGS, zaro@ufrgs.br
}

\begin{abstract}
This paper presents a technology platform as a resource for the teaching of control engineering. The platform in question combines the educational potential of software of design and simulation of control systems - MATLAB ${ }^{\circledR}$ - and the connection between this software and a pilot distillation column. The research hypothesis is that the use of technology platforms such as this help to develop aspects not only stimulated with simulation. Therefore, an experiment based on the constructivist and socio-interactionist theory has been designed to detect students development and to capture their impressions of the platform. The results are presented as graphs and impressions reports of the teacher.
\end{abstract}

Keywords: technological platform, control systems, constructivist theory.

\section{Introdução}

A ênfase no ensino de técnicas no lugar de conceitos resulta em um rápido esquecimento por parte dos alunos. O ensino da teoria desvinculado dos aspectos práticos não prepara adequadamente o aluno para o exercício da profissão. (Ribas et al. 1998 citado por Vallim et al. 2000).

Atualmente no ensino de engenharia os métodos utilizados pelos professores não contribuem de forma significativa para o desenvolvimento das habilidades $\mathrm{e}$ competências necessárias ao profissional, sobretudo em um curso de engenharia de controle e automação. 
As teorias selecionadas para a concepção didático-pedagógica desta plataforma têm, como principal argumento, a tentativa de aproximação do ensino de engenharia, tipicamente instrucionista, ao paradigma mais adequado para a construção do saber, do conhecimento e das competências inerentes a este profissional, em contraponto as teorias positivistas e comportamentalistas dominantes neste segmento da educação de nível superior. Dotada de um software de projeto e simulação de sistemas de controle e ainda de integração entre o software de simulação e um sistema real em escala, a referida plataforma, proporciona ao aluno o ambiente favorável ao desenvolvimento de competências e habilidades necessárias a sua profissão.

Segundo Schinaid et al. (2003) as recomendações da ABET-Accreditation Board for Engineering and Technology, instituição norte-americana que procura estabelecer critérios de qualidade específicos para cada habilitação, os cursos de graduação devem estimular a capacidade para aplicar conhecimento de matemática, ciências e engenharia; projetar e conduzir experimentos; analisar e interpretar resultados; projetar um sistema, componente ou processo para atender a determinados requisitos; atuar em equipes multidisciplinares; identificar, formular e resolver problemas de engenharia; poder compreender a natureza da ética e da responsabilidade profissional; comunicar-se efetivamente (por escrito e oralmente); entender o impacto das soluções da engenharia no contexto social e ambiental; buscar a aprendizagem permanente; e ainda usar técnicas e ferramentas modernas para o exercício da prática da engenharia.

Com o suporte computacional apropriado este profissional, ainda em formação, tem acesso a situações-problema comuns em seu ambiente profissional desde que em consonância a este recurso seja elaborada uma estratégia didático-pedagógica por parte do professor que favoreça a produção/autoria e o desenvolvimento do educando. Para tanto, certos aspectos não podem deixar de ser destacados como, por exemplo, o processo avaliativo.

É comum em cursos de engenharia que os professores, exímios transmissores de conhecimento - como se o conhecimento pudesse ser transmitido e não construído entendem como avaliação um processo de confronto entre as respostas do professor e as respostas do aluno, buscando uma medida de aproximação entre as duas para gerar uma provável nota ou conceito que representa todo o aprendizado do indivíduo ao longo do semestre letivo.

Acompanhamento do processo de construção de conhecimento implica favorecer o desenvolvimento do aluno, orientá-lo nas tarefas, oferecer-lhe novas leituras ou explicações, sugerir-lhe investigações, proporcionar-lhe vivências enriquecedoras e favorecedoras à sua ampliação do saber [Hoffmann 1994].

No contexto da formação fundada em paradigmas que se constituem a partir do construtivismo, das pedagogias críticodialógicas e sócio-interacionistas, a avaliação educacional não é algo que está dissociada do grande conjunto processual que configura a educação formal escolar e universitária. Ela é intrínseca à prática educativa, ao ensino e à aprendizagem [Ahlert 2002].

Todavia, o processo de avaliação da aprendizagem deve ser contínuo e presente ao longo de todo o processo de ensino-aprendizagem, uma avaliação estanque como uma prova não será capaz de representar todo o conhecimento construído nas estruturas cognitivas do aluno. 
O trabalho está estruturado da seguinte forma: na seção 2 é apresentado um breve estado da arte da utilização de plataformas tecnológicas semelhantes, na seção 3 é apresentada a plataforma tecnológica proposta, destacando seus principais componentes constituintes. Na seção 4 apresenta-se a proposta de caracterização de um experimento didático-pedagógico concebido e na seção 5 os resultados do estudo de caso. Na seção seis as considerações finais do trabalho.

\section{Um breve estado da arte}

$\mathrm{O}$ estado da arte que congrega diversos estudos relacionados à plataforma proposta pode ser abordado de forma bastante resumida nos próximos parágrafos. É importante destacar que apesar da similaridade da plataforma proposta com os diversos laboratórios remotos mencionados nos trabalhos que se seguem, este trabalho tem como intuito utilizar o recurso tecnológico como facilitador para um experimento didáticopedagógico que objetiva investigar a importância desta plataforma para o ensino de engenharia de controle e automação.

Aktan, et al. (1996) baseiam-se no paradigma do aprendizado à distância, no qual aluno, professor e equipamento estão geograficamente separados, para desenvolver um laboratório de acesso remoto em tempo real para ensino de engenharia de controle. Todo o aparato implementado permite que os alunos possam desenvolver, compilar e depurar os programas a distância e em um segundo momento, realizar experimentos online para controle de um manipulador robótico.

Liou, et al. (1999) utilizam o software Labview para controle de sistemas elétricos como circuitos RLC e motores elétricos. Através da Internet acessam parâmetros do Labview, permitindo que o usuário possa interagir com os sistemas nele simulados.

Schmid e Ali (2000) desenvolvem um sistema baseado em Internet para ensino de engenharia de controle. Este sistema permite visualização e simulação de sistemas dinâmicos de forma mais abrangente devido a utilização de um web-browser padrão. Utilizam para animação dos sistemas dinâmicos a linguagem VRML (Virtual Reality Modelling Language), além de contar com estes recursos o curso virtual é composto por tutoriais, exercícios e experimentos virtuais.

Clume e Gomes (2001) apresentam um sistema para controle remoto de processos em tempo real através da Internet, seguindo uma arquitetura cliente-servidor. Uma interface de projeto e sintonia de controladores fuzzy é disponibilizada para o usuário. $\mathrm{O}$ equipamento acessado remotamente é um pêndulo. Testes foram realizados entre as universidades UFJF (Universidade Federal de Juiz de Fora) e UFSC (Universidade Federal de Santa Catarina) para validar a capacidade de acesso remoto do sistema.

Zeilmann (2002) apresenta uma proposta de controle supervisão e monitoramento de processos industriais pela Internet. Apresenta o trabalho sob a luz dos três contextos envolvidos: a planta industrial, o servidor e o cliente. Através da integração de tecnologias web e redes fieldbus, propicia acesso remoto por parte dos usuários aos dispositivos que se comunicam via protocolo Foundation Fieldbus. Apresenta avanço em relação a outros laboratórios remotos pela proximidade entre a planta piloto utilizada e as encontradas na indústria.

Casini, et al. (2003) desenvolvem um laboratório para o ensino remoto de técnicas de controle de processos. Os módulos de controle disponíveis no laboratório são: controle de posição, controle de velocidade, ambos de um motor DC, nível de tanque, e 
simulador de helicóptero. Foca na competição entre os usuários e seus algoritmos de controle, criando um ranking para os que desenvolvem algoritmos com melhor desempenho.

Dormido, et al. (2008) apresentam um laboratório on-line para experimentos em um sistema não linear multi-variável, três tanques acoplados, permitindo que estudantes possam aprender na prática, aspectos fundamentais de controle de processos. Através da integração das ferramentas: EJS (Easy Java Simulations) e eMersion - ferramenta colaborativa de experimentos on-line, os envolvidos podem ter uma maior flexibilidade na realização dos experimentos.

Partindo deste cenário, é perceptível um grande enfoque na verificação da validade da ferramenta apenas de forma quantitativa e no processo de construção destas plataformas tecnológicas. O propósito deste trabalho é observar a evolução do aluno durante a utilização da ferramenta, o que leva basicamente todo o semestre letivo, lançando mão de dois instrumentos de verificação: um questionário para os alunos e os registros das impressões do professor.

\section{A plataforma tecnológica proposta}

A elaboração de uma plataforma tecnológica para o ensino de engenharia de controle passa pela utilização de recursos computacionais e tecnológicos existentes e disponíveis no laboratório de automação industrial do Instituto Federal A. Estes recursos, ora explorados separadamente, agora passam a integrar um ambiente de projeto, simulação, implementação e testes para estratégias de controle avançado.

Diferente dos muitos laboratórios acessados remotamente disponíveis em instituições de ensino pelo mundo, o propósito deste laboratório é servir como base para estudos de alunos matriculados em um curso regular e presencial, deixando claro que a intenção, pelo menos não inicial, deste trabalho não é investigar as vantagens destes laboratórios para educação à distância.

A plataforma concebida é dotada de três elementos constituintes básicos:

1. Sistema real, representado na forma da coluna de destilação piloto.

2. Software de projeto e simulação de sistemas de controle MATLAB/SIMULINK®.

3. Integração do software acima relacionado com o sistema real em questão, através de um sistema de medição e aquisição de dados industrial - Foundation Fieldbus (SYSCON®) e os servidores OPC que integram o pacote SYSTEM302 do fabricante Smar®.

A opção por softwares proprietários se deve ao fato da não existência de softwares equivalentes para as funções inerentes à aplicação, salientando que todos os softwares utilizados são devidamente licenciados. A Figura 1 apresenta na forma de diagrama os elementos constituintes da plataforma. 


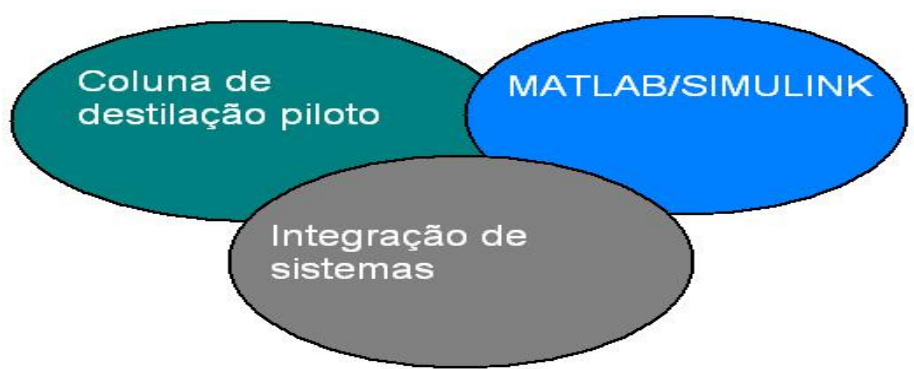

Figura 1. Componentes da plataforma tecnológica.

\subsection{A coluna de destilação}

Em diversos laboratórios do curso de automação do Instituto Federal A existem protótipos de plantas e processos industriais. Por meio de sistemas de medição e aquisição de dados similares aos utilizados em ambiente industrial, estes protótipos permitem aos alunos a interação com o equipamento e aprendizado de técnicas de identificação e controle de processos que são utilizadas por engenheiros de controle e automação. Dentre os laboratórios existentes o que mais se aproxima de um processo industrial nos quesitos sistema de instrumentação e capacidade produtiva, é a coluna de destilação piloto. Uma imagem da coluna de destilação pode ser vista na Figura 2.

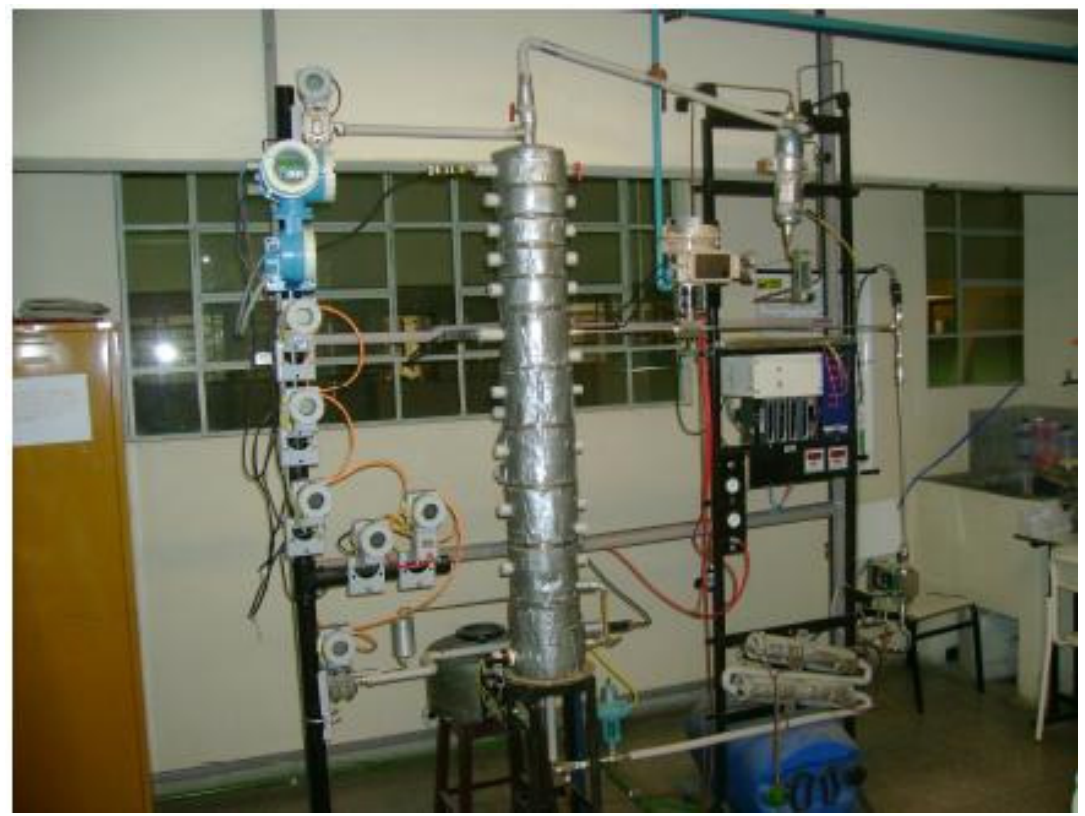

Figura 2. Coluna de destilação piloto.

\subsection{Integração de softwares de comunicação}

Esta coluna possui um sistema de supervisão e controle bem próximo da realidade encontrada nas indústrias de transformação que baseiam seus processos na destilação, destacam-se usinas de açúcar e álcool e refinarias de petróleo.

Por meio de uma integração entre o SYSCON® e seus servidores OPC instalados é possível realizar o acesso aos parâmetros dos instrumentos ligados à planta, desta forma possibilitando a medição de variáveis e também atuação em elementos finais de controle como as válvulas de controle de vazão. Esta disponibilidade de dados será utilizada pelo 
MATLAB® para o último nível de integração permitindo o controle da coluna de destilação.

\subsection{O software de projeto e simulação}

O MATLAB ${ }^{\circledR}$ possui uma grande aceitação por parte de engenheiros por conter toolboxes para diversas áreas do conhecimento científico, sobretudo nas engenharias. Em termos de engenharia de controle ele possui um ambiente gráfico que trabalha com o conceito de diagrama de blocos e fluxos de sinal para o projeto e simulação de sistemas de controle conhecido como SIMULINK®. A partir da versão 7 o MATLAB® passou a contar com um toolbox específico para comunicação OPC que é o protocolo de transferência dinâmica mais utilizado na indústria, proporcionando então a integração de um software utilizado na academia à equipamentos de porte industrial, como os instrumentos da coluna de destilação.

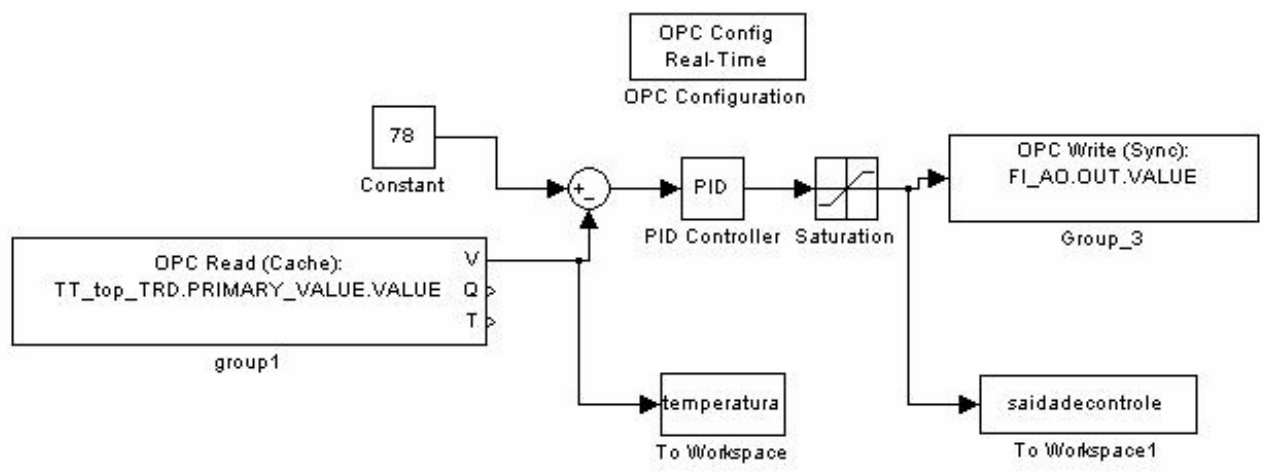

Figura 3. Sistema de controle com comunicação OPC.

A Figura 3 apresenta uma tela do SIMULINK® com um sistema de controle implementado acessando parâmetros dos instrumentos a partir de blocos de comunicação OPC. Os sistemas de controle projetados e simulados pelos alunos podem então ser testados no sistema real.

\section{Caracterização do experimento}

O experimento didático-pedagógico foi realizado no âmbito do Instituto Federal A e utilizou como campo empírico os alunos do $8^{\circ}$ período do curso superior de engenharia de controle e automação, mais especificamente circunscrito aos alunos matriculados na disciplina de controle avançado.

Certos aspectos devem ser observados ao longo do semestre letivo para que, no final do curso, seja possível a compilação e análise dos resultados sob a luz das teorias envolvidas. Levando em conta tais aspectos, na caracterização do experimento didáticopedagógico são apresentadas as etapas constituintes.

Dentre estas etapas, pode-se destacar:

1. Estruturação física do laboratório. Os alunos ficam dispostos em computadores sobre bancadas com processador de dois núcleos de 2.4 $\mathrm{GHz}$ com Cache de 2MB, 2GB de RAM, disco rígido de $120 \mathrm{~GB}$ padrão SATA, monitor LCD TFT 19" Widescreen com base conforme regras ABNT, teclado ABNT2. Estes PC's estão conectados a um Hub juntamente com a DFI (Device Fieldbus Interface) permitindo o acesso 
de todos os computadores à planta. Licença para o MATLAB® versão R2006a para até 20 máquinas na rede.

2. Verificação das impressões relativas à evolução dos alunos na forma de registros diários feitos pelo professor da disciplina. O extrato destes registros é apresentado na seção 5.

3. Registro das impressões dos alunos com relação à plataforma. Foi utilizada uma versão modificada do instrumento SERVQUAL, originário da verificação da qualidade de serviços, mais informações serão apresentadas na seção 5.

Esta proposta de experimento delineia que ao longo das aulas o professor desempenho o papel de idealizador e problematizador para o aluno, bem como um possível parceiro no processo de ensino-aprendizagem ora como um auxiliador do aluno, ora como instigador de novas investigações.

As etapas da metodologia sugerida para a caracterização e execução do experimento estão galgadas na premissa de que, não é correto enxergar os processo de ensinoaprendizagem como um processo estanque que inicia em certo ponto e termina em outro com uma avaliação de conceitos, mas sim como um processo contínuo que promove evolução diária e que necessita de períodos de reflexão entre processos de assimilação e acomodação sequienciais e resultando na tão destacada abstração reflexionante de Piaget (2007) fruto não apenas de ações do indivíduo, mas da coordenação de ações.

Dentro desta realidade a plataforma tecnológica proposta se insere como um ambiente no qual os alunos poderão projetar simular e implementar os sistemas de controle avançado concebidos e testar o seu desempenho sobre a coluna de destilação piloto.

\section{Resultados}

Os resultados do experimento foram divididos em duas partes, uma relativa aos relatos das impressões do professor em sala de aula e a outra relativa à verificação da lacuna existente entre a expectativa e a percepção dos alunos ao utilizarem a plataforma computacional proposta.

Parasuraman et al. (1985) apresentaram a metodologia que utilizaram para a criação da escala SERVQUAL. Trata-se de um processo iterativo de avaliação da qualidade de cinco empresas do ramo de serviços na década de 80 nos EUA e subseqüente verificação da consistência interna dos questionários através do coeficiente alpha de Cronbach ${ }^{1}$. A partir de cento e cinquenta itens os autores chegam a um questionário final com apenas vinte e dois itens, podendo estes itens ainda ser reduzidos. Neste trabalho foram elaborados apenas dez itens, dando origem ao questionário aplicado aos alunos.

A escolha pela escala SERVQUAL neste trabalho é motivada pela facilidade em quantificar valores subjetivos e impressões dos clientes, neste caso os alunos, e sua importante capacidade de demonstrar a lacuna entre expectativa e percepção tornando um pouco mais tangíveis os aspectos verificados.

Os alunos avaliaram cada um dos dez itens em relação à expectativa e percepção, para isso eles tiveram que enquadrar os itens em uma das posições da escala numérica

\footnotetext{
${ }^{1}$ Alpha de Cronbach é um coeficiente utilizado como medida de consistência interna de questionários.

V. $7 \mathrm{~N}^{\mathrm{o}}$ 3, dezembro, 2009
} 
composta por sete posições conhecida como escala de Likert ${ }^{2}$ em função do seu nível de concordância com afirmação apresentada. De acordo com o trecho retirado do questionário:

A plataforma tecnológica utilizada facilitou nas tarefas a serem realizadas.

\begin{tabular}{|l|l|l|l|l|l|l|}
\hline 1 & 2 & 3 & 4 & 5 & 6 & 7 \\
\hline
\end{tabular}

Outros exemplos de afirmações utilizadas podem ser apresentados como: A plataforma tecnológica utilizada é indispensável para realização das tarefas; A plataforma foi relevante para a sedimentação dos conceitos trabalhados em sala de aula; $A$ plataforma tecnológica auxilia no desenvolvimento de competências necessárias à sua formação profissional. Ao todo foram utilizadas dez afirmações. A partir dos dados coletados por meio do questionário foi possível estabelecer graficamente a diferença entre expectativa e percepção, em termos de frequência das respostas, dando origem ao gráfico da Figura 4.

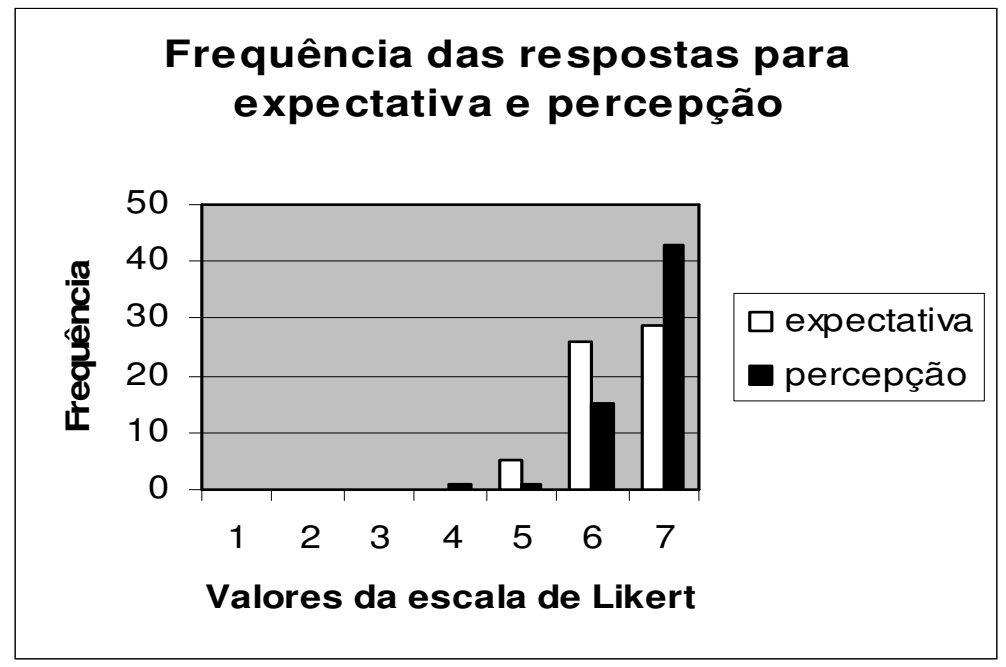

Figura 4. Gráfico de frequiência das respostas para a expectativa e a percepção.

Percebe-se no gráfico da Figura 4 uma frequência bastante elevada para o valor sete no aspecto da percepção. Pode-se concluir a partir disso que em grande parte dos itens do questionário os alunos concordam fortemente com a afirmação proposta. De outra forma a frequência não menos elevada dos valores seis e sete para a expectativa sugere que os alunos possuíam uma boa expectativa quanto à ferramenta, que é confirmada ou superada positivamente na percepção.

O acompanhamento dos alunos ao longo do semestre letivo foi realizado de forma diária pelo professor da disciplina de controle avançado, apenas o extrato de suas impressões será sumariado neste artigo, dando enfoque às impressões mais relevantes. A turma é composta de oito alunos do oitavo período do curso de engenharia de controle e automação. Todos os trechos com recuo a seguir se referem a falas do professor da disciplina.

De forma geral os alunos obtiveram um excelente desempenho na disciplina. Desempenho este que foi verificado ao final do semestre,

\footnotetext{
${ }^{2}$ Escala de Likert é uma escala de cinco ou sete valores utilizada para quantificar aspectos subjetivos. 
todavia o conhecimento foi sendo construído a cada interação em sala de aula.

Analisando o comentário do professor é possível verificar que a evolução dos alunos durante o semestre foi bastante evidente, refletindo nos resultados de desempenho ao final do semestre letivo.

Os alunos inicialmente tiveram aulas expositivas de fundamentação teórica sobre as principais técnicas de controle avançado.

Em certo ponto os alunos obtiveram explanações sobre a utilização da plataforma tecnológica proposta para desenvolvimento $e$ aplicação de sistemas de controle avançado.

Uma fundamentação teórica foi necessária para municiar os alunos para que possam, mais facilmente, alcançar os objetivos que são propostos em etapas subseqüientes.

A partir daí, os alunos foram incentivados a trabalhar por projetos $e$ a solucionar problemas específicos de controle utilizando a plataforma. Em diversas aulas os alunos apresentaram bastante iniciativa e se auxiliavam demonstrando capacidade de trabalho em equipe.

Ao trabalhar com um novo modelo de aula que favoreceu a participação e produção, e estimulados a trabalhar por projetos, se estabeleceu um clima propício para o desenvolvimento de competências bastante valorizadas como o trabalho em equipe.

Por meio de um trabalho de fim de período no qual os alunos tiveram que projetar, implementar e testar um sistema de controle avançado utilizando a plataforma proposta, foi possível perceber que eles já possuíam desenvoltura na manipulação da ferramenta. Ao findar do semestre os alunos apresentaram seus projetos e sistemas de controle funcionando de acordo com as especificações.

É possível verificar que eles desenvolveram a principal competência necessária a um engenheiro de controle e automação, ou seja, desenvolvimento e aplicação de uma estratégia de controle para processos industriais.

\section{Considerações Finais}

Percebe-se que o ambiente proposto visa proporcionar aos alunos um ambiente favorável para o desenvolvimento das competências e habilidades necessárias para um engenheiro de controle e automação, como os apresentados em Schinaid et al. (2003), desta forma atinge o objetivo a partir da análise dos resultados apresentados.

A concepção didático-pedagógica do experimento e a análise dos resultados foram desenvolvidas no sentido de aproximação das teorias construtivistas, principalmente nos aspectos de fundamentação da proposta e instrumentos utilizados para obtenção dos resultados. Os resultados obtidos pela aplicação dos instrumentos foram considerados satisfatórios para um estudo piloto. Pode-se verificar tal afirmação a partir da apresentação dos resultados de forma gráfica para a aceitação da plataforma por parte dos alunos e também na forma dos relatos do professor. 
Sugere-se para trabalhos futuros a aplicação em outras turmas de controle avançado e elaboração/utilização de novos instrumentos para acompanhamento da evolução dos alunos durante o semestre letivo como, por exemplo, relatório diário.

\section{Referências bibliográficas}

AHLERT, A. A avaliação como um processo interno da prática pedagógica. Caderno de Educação Física: estudos e reflexões, Marechal Cândido Rondon, v. 4, n. 8, p. 119-125, 2002.

AKTAN, B., BOHUS, C. A., CROWL, L. A., SHOR, M. H. Distance learning applied to control engineering laboratories. IEEE Transactions on Education, New York, v.39, n.3, p.320-326, Aug., 1996.

CASINI, M., PRATTICHIZZO, D., VICINO, A. E-learning by remote laboratories: a new tool for control education. In: IFAC Symposium on advances in control education, Finland. University of Oulu. p.95-100, 2003.

CLUME, M. F., GOMES, F. J. NetControl: Ambiente para controle de processos em tempo real. VII Encontro de Ensino em Engenharia. Faculdade de Engenharia da Universidade Federal de Juiz de Fora, 2001.

DORMIDO, R., VARGAS, H., DURO, N., SÁNCHEZ, J., DORMIDO-CANTO, S., FARIAS, G., ESQUEMBRE, F., DORMIDO, S. Development of a web-based control laboratory for automation technicians: the three-tank system. IEEE Transactions on Education, New York, v.51, n.1, p.35-44, Feb, 2008.

HOFFMANN, J. Avaliação mediadora: uma relação dialógica na construção do conhecimento. 1994. Disponível em http://www.crmariocovas.sp.gov.br. Acesso em: 20 jul. 2005.

LIOU, S., SOELAEMAN, H., LEUNG, P. Distance Learning Power Engineering Laboratory. Submitted to IEEE Computer Applications in Power, 1999.

PARASURAMAN, A., ZEITHAM, V. A., BERRY, L. L. A Conceptual Model of Service Quality and Its Implications for Future Research, Journal of Marketing, p. 41-50, 1985.

PIAGET, J. Epistemologia Genética, São Paulo: Martins Fontes, 2007 (Col. Psicologia e Pedagogia).

SCHIMID, C.; ALI, A. A web-based system for control engineering education. In: Proceedings of the American Control Conference, Chicago: AACC. p.34633990, 2000.

SCHNAID, F., TIMM, M. I., ZARO, M. A. Considerações sobre uso de modelo construtivista no ensino de Engenharia - disciplina de projeto, com graduandos e mestrandos. RENOTE - Revista Novas Tecnologias na Educação, Porto Alegre:UFRGS, v. 1, n. 1, 2003.

VALLIM, M. B. R, FARINES, J., CURY, J. E. R., Em direção à melhoria do ensino na área tecnológica: a experiência de uma disciplina de introdução à engenharia de controle e automação, In: Anais do XIII Congresso Brasileiro de Automática - CBA 2000, Florianópolis - SC - Brasil.

ZEILMANN, R. P. Uma estratégia para controle e supervisão de processos industriais via Internet. Dissertação, mestrado em Engenharia Elétrica, UFRGS Universidade Federal do Rio Grande do Sul, 2002. 